\title{
Evolution and development of the slow, dusty nova, V1280 Scorpii (Nova Sco 2007a)
}

\section{Andrew Helton ${ }^{a}$, William Vacca ${ }^{a}$, Nye Evans ${ }^{b}$, Charles E. Woodward ${ }^{c}$, Robert D.} Gehrz $^{c}$, Frederick Walter ${ }^{d}$, and Dinesh Shenoy ${ }^{c}$

${ }^{a}$ SOFIA Science Center, USRA, NASA Ames Research Center MS N232-12, Moffett Field, CA 94035, USA

${ }^{b}$ Astrophysics Group, Keele University

Keele, Staffordshire, ST5 5BG, UK

${ }^{c}$ Minnesota Institute for Astrophysics, University of Minnesota - Twin Cities

116 Church St. S.E., Minneapolis, MN 55455, USA

${ }^{d}$ Dept. of Physics \& Astronomy, University of New York at Stony Brook

Stony Brook, NY 11794, USA

E-mail: aheltonesofia.usra.edu, wvaccaesofia.usra.edu, ae@astro.keele.ac.uk, chelsea@astro.umn.edu, gehrzeastro.umn.edu, fwalter@mail.astro.sunysb.edu, shenoy@astro.umn.edu

\begin{abstract}
The classical nova V1280 Sco erupted in February 2007 and was one of the brightest novae of the past decade. The system began producing an optically thick dust shell around 10 days after eruption, much earlier than is typical for dust producing novae. The dust condensation event lasted more than 100 days and preceded a very slow, protracted evolution of the ejecta. Early nearand mid-IR observations indicated that hydrocarbons were likely present. These characteristics made V1280 Sco an excellent laboratory in which to examine the evolution and development of dusty novae in general and specifically to explore the processes of dust condensation, growth, and destruction. Here, we discuss the history of this fascinating nova and its implications for our understanding of dust formation and processing, with a particular emphasis on the role of hydrocarbon species. We also present near-IR data from SpeX on the IRTF, which demonstrates that the ejecta are likely still composed of optically thick clumps and that aliphatics are no longer significant contributors to the hydrocarbon population.
\end{abstract}

The Life Cycle of Dust in the Universe: Observations, Theory, and Laboratory Experiments - LCDU 2013, 18-22 November 2013

Taipei, Taiwan 


\section{Classical novae}

A classical nova $(\mathrm{CN})$ consists of a binary system, in which a white dwarf (WD) accretes material onto its surface from a main sequence companion, typically through Roche-lobe overflow. After a period of $\geq 10^{3}$ years, the lower layers of the accreted material reach a high enough temperature and density to trigger a thermonuclear runaway throughout the WD envelope [1], culminating in the ejection of much, if not all, of the accreted material $\left(10^{-6}-10^{-4} M_{\odot}\right)$ at velocities ranging from hundreds to thousands of $\mathrm{km} \mathrm{s}^{-1}$. The magnitude of the outburst varies greatly, but is typically between the range of $8<\mathrm{m}_{\mathrm{V}}<14 \mathrm{mag}$ [1].

$\mathrm{CNe}$ frequently produce dust in their ejecta. The fraction of $\mathrm{CNe}$ that produce dust is not well known, but is thought to range from $\sim 20 \%$ [2] to $\sim 40 \%$ [3]. Dust production in the dustiest $\mathrm{CNe}$ manifests itself in the visual light curve by a strong extinction event often of $>5$ mag developing over the course of only a few days (Fig. 1) and accompanied by a sharp rise in the infrared luminosity as the optical emission is reprocessed by the dust [4].

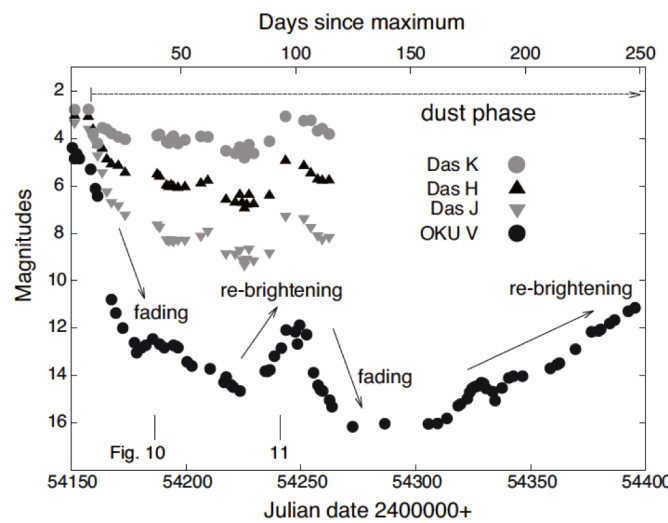

Figure 1: Light curve of V1280 Sco [5]. After the initial period of dust condensation and the associated optical extinction, the system underwent a brief re-brightening. The fact that the IR brightness increased simultaneously during this second event indicates that it was not simply the result of the exposure of the underlying pseudophotosphere, but was a second, possibly ionization induced, dust formation event [6].

\section{Dust species in classical novae}

$\mathrm{CNe}$ are unusual among dust producing systems in that they occasionally produce multiple species of dust, such as silicates, amorphous carbon, and hydrocarbons, during a single dust formation event (e.g., QV Vul [7]) with a grain size distribution reaching up to $\sim 1 \mu \mathrm{m}$ in size [4]. The canonical picture of dust production begins with the formation of simple molecules, including $\mathrm{CO}$, $\mathrm{CN}$, and $\mathrm{H}_{2}$, which may then act as the nucleation sites on which dust grains condense [8]. The role of larger, complex hydrocarbon molecules in the growth of more substantial grains is unclear, but their presence is unmistakable.

Hydrocarbons in most astronomical sources have been identified with polycyclic aromatic hydrocarbons (PAHs [9]). These PAHs consistently exhibit a variety of signatures, including a number of features between 5 and $20 \mu \mathrm{m}$ with wavelengths and profiles that vary somewhat due to differences in their local environmental conditions $[10,11]$. However, hydrocarbons in the ejecta of $\mathrm{CNe}$ exhibit broad, complex plateaus as well as discrete features with central wavelengths and relative intensities inconsistent with other astronomical PAHs [12, 13]. Instead, the features are consistent with more complex species such as hydrogenated amorphous carbon (HACs [14, 12, 13, 15]). 


\section{V1280 Sco - Evolution}

V1280 Sco went into outburst on 2007 February 4. This nova turned out to be unique in many respects. The onset of dust formation occurred only 11-12 days after maximum, the earliest a dust condensation event that has ever been observed in a nova (Fig. 1). Recovery of the light curve after the initial dust extinction event took more than 250 days. P-Cygni profiles were still observed on emission lines many years after the initial outburst, leading several authors to conclude that the system experienced an extremely long period of mass loss. This corresponds well with the exceptionally slow evolution of the emission spectrum, the slowest nova development on record.

Near day 110, the light curve underwent a brief period of re-brightening. Though it is tempting to attribute this to clearing of the ejecta and exposure of the underlying pseudo-photosphere, the corresponding increase in IR flux instead suggests a second period of mass loss and additional dust production [5]. This is supported by modeling of the ejecta performed by Chesneau et al. [6] that suggested a second shell of material was ejected around that time. Those authors suggested that the subsequent dust formation could, in fact, have been ionization induced, as hypothesized by Shore \& Gehrz[16] and contrary to standard models for dust production in $\mathrm{CNe}$ [8].

\section{Recent observations of V1280 Sco}

The unique character of V1280 Sco inspired us to take advantage of its slow development to examine the evolution of the dust formed in its ejecta as it was being cleared and exposed to the hot, underlying white dwarf. Our goals were to: 1) measure the conditions within the dusty environment (e.g., temperatures, densities, etc.); 2) estimate the dust mass; and 3) determine the composition and mineralogy of the dust, which includes 4) the relative contribution of aromatic to aliphatic hydrocarbons and 5) the identity of the carriers of any hydrocarbon features that may be present. We planned a series of near- and mid-IR spectroscopic observations using the FORCAST (MIR) and FLITECAM (NIR) instruments on SOFIA with supplementary NIR observations from SpeX on the IRTF in order to construct a meaningful interpretation of the dust grain formation pathways and gain insight into the role that hydrocarbon chemistry plays in the condensation, growth, and destruction of dust. Due to scheduling constraints, we were unable to obtain the SOFIA data. Nevertheless, we did acquire three epochs of SpeX IRTF observations, with which we intend to assess the conditions in the ejecta at late times and to gain insight into the relative contributions of aromatics to aliphatics (Figs. $2 \& 3$ ).

The first epoch of SpeX data on V1280 Sco, acquired more than 2000 days after outburst is shown in Figure 2. The exceedingly slow evolution is apparent from the persistence of strong P-Cyg absorption profiles on the He I lines and the weak P-Cyg profiles on the $\mathrm{H}$ recombination lines. The persistence of neutral species, such as Mg I, C I, and Na I support the hypothesis that the ejecta are clumpy, while the $\mathrm{C}$ I lines suggest a prevalence of carbonaceous dust. At these late times, the aliphatic feature from 3.4-3.55 $\mu \mathrm{m}$ is very weak relative to the aromatic feature at 3.3 $\mu \mathrm{m}$ and shows little variation between the three epochs of SpeX data (Fig. 3). Hence, even if aliphatics accounted for a significant fraction of the hydrocarbon species early on, at late times, their contribution is likely of only secondary importance. 

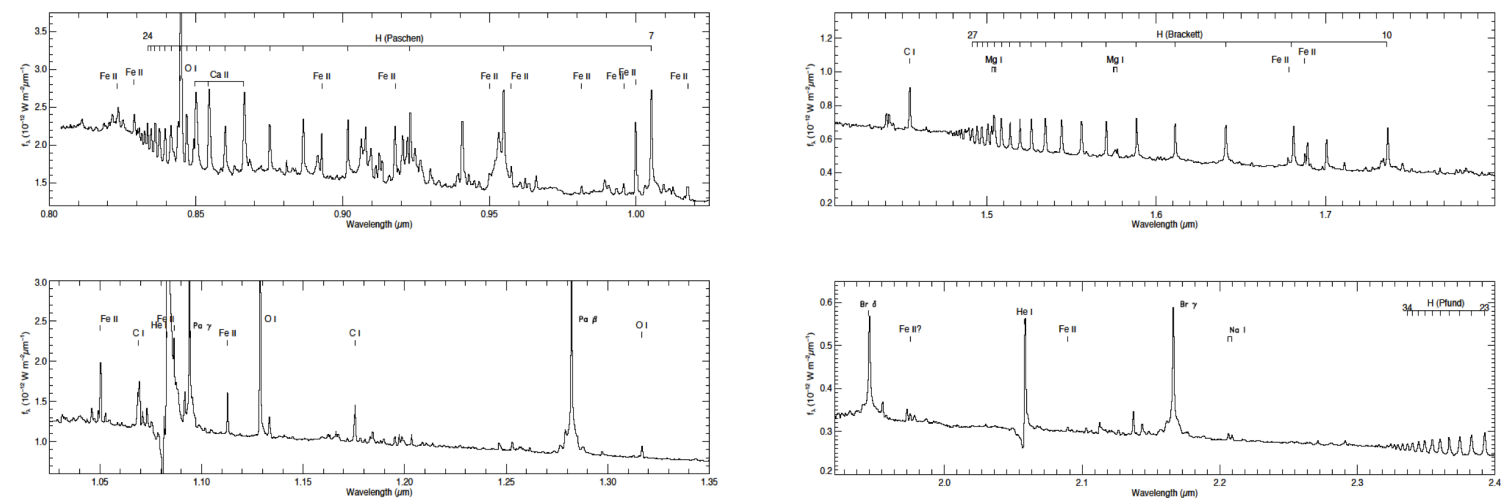

Figure 2: $0.8-2.4 \mu \mathrm{m}$ spectrum of V1280 Sco obtained with SpeX on IRTF more than 2000 days after outburst.

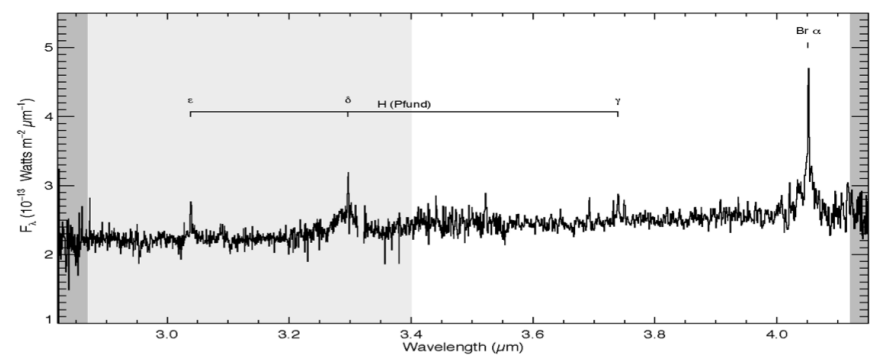

Figure 3: Epoch $1 \mathrm{SpeX}$ spectrum of the $3.3-3.5 \mu$ m hydrocarbon complex.

\section{References}

[1] Warner, B. 2008, in Classical Novae, $2^{\text {nd }}$ Edition, eds.: M. F. Bode \& A. Evans, (Cambridge Univ. Press: Cambridge)

[2] Strope, R. J., Schaefer, B. E., \& Henden, A. A. 2010, AJ, 140, 34

[3] Helton, L. A. 2010, Ph.D. Thesis

[4] Gehrz, R. D. 2008, in Classical Novae, $2^{\text {nd }}$ Edition, eds.: M. F. Bode \& A. Evans, (Cambridge Univ. Press: Cambridge)

[5] Naito, H., Mizoguchi, S., Arai, A., et al. 2012, A\&A, 543, A86

[6] Chesneau, O., Banerjee, D. P. K., Millour, F., et al. 2008, A\&A, 487, 223

[7] Gehrz, R. D. 1992, ApJ, 400, 671

[8] Evans, A., \& Rawlings, J. M. C. 2008, in Classical Novae, $2^{\text {nd }}$ Edition, eds.: M. F. Bode \& A. Evans, (Cambridge Univ. Press: Cambridge)

[9] Allamandola, L. J., Tielens, A. G. G. M., \& Barker, J. R. 1989, ApJS, 71, 733

[10] Peeters, E., Hony, S., Van Kerckhoven, C., et al. 2002, A\&A, 390, 1089

[11] van Diedenhoven, B., Peeters, E., Van Kerckhoven, C., et al. 2004, ApJ, 611, 928

[12] Evans, A., Gehrz, R. D., Woodward, C. E., et al. 2010, MNRAS, 406, 85

[13] Helton, L. A., Evans, A., Woodward, C. E., \& Gehrz, R. D. 2011, EAS Publications Series, 46, 407

[14] Duley, W. W., \& Williams, D. A. 1981, MNRAS, 196, 269 
[15] Kwok, S., \& Zhang, Y. 2011, Nature, 479, 80

[16] Shore, S. N., \& Gehrz, R. D. 2004, A\&A, 417, 695 